\title{
Design and simulation of equilateral triangular microstrip antenna using particle swarm optimization (PSO) and advanced particle swarm optimization (APSO)
}

\author{
PRABAL PRATAP $^{1, *}$, RAVINDER SINGH BHATIA ${ }^{2}$ and BINOD KUMAR ${ }^{3}$ \\ ${ }^{1}$ National Institute of Technology Kurukshetra, Kurukshetra, Haryana 136119, India \\ ${ }^{2}$ Department of Electrical Engineering, National Institute of Technology Kurukshetra, Kurukshetra, \\ Haryana 136119, India \\ ${ }^{3}$ Department of Electronics Engineering, Ambedkar Institute of Technology, Delhi 110031, India \\ e-mail: prabalpr@gmail.com; rsibhatia@yahoo.co.in; bkkanaujia@yahoo.co.in
}

MS received 13 November 2014; revised 19 December 2015; accepted 13 February 2016

\begin{abstract}
In this paper a new design is proposed in microstrip antenna family. In this paper, a review design of microstrip antenna design using particle swarm optimization (PSO) and advanced particle swarm optimization (APSO) has been presented which optimizes the parameters and both results are compared. This technique helps antenna engineers to design, analyze, and simulate antenna efficiently and effectively. An advanced PSO driven antenna has been developed to calculate resonant frequency of slit-cut stacked equilateral triangular microstrip antenna. The paper presents simplicity, accuracy and comparison of result between PSO and APSO.
\end{abstract}

Keywords. Microstrip antenna; bandwidth; PSO; advanced PSO; resonant frequency.

\section{Introduction}

The microstrip antenna has been widely used in satellite, planner antenna in missile technology and mobile communication because of its attractive features and low fabrication cost. Microstrip antenna is like a parallel plate capacitors. Microstrip antenna has a radiating patch on one side of dielectric substrate which has ground plane on either side. Electrical characteristics of microstrip antenna starting from rectangular shape patch, when antenna is operating in transmitting mode, the antenna is driven with a voltage between probe feed and ground plane. This excites current on the patch, and the vertical electric field between the patch and ground plane. Deschamps developed the planar antennas integrated with transmission lines [1, 2]. There are various shapes of microstrip patches, such as rectangular, circular, triangular, semicircular, sectoral and annular, etc., are successfully used as radiating antenna elements. Triangular microstrip antenna has advantages for designer to reduce coupling between adjacent elements of array. Equilateral triangular microstrip antenna (ETMA) is more directive antenna with compactness in size. Lots of techniques have been used for size reduction and bandwidth enhancement like slot loading techniques and slit cut in patch. Conventional ETMA was studied in [3-7]. Different design techniques are successfully implemented to

*For correspondence improve the performance of microstrip antenna. Antenna suffers from serious drawbacks like narrow bandwidth, low gain, single mode frequency, and poor radiation performance. Choice of feed position is important parameter and efficiency. Advanced PSO is used to quarter these challenges. In advanced PSO, the solution space is enlarged in the optimization by relaxing the constraint imposed by previous studies that finding of local best and global best of mechanism of displacement and velocity particles. Advanced particle swarm optimization (APSO) is applied to improve the quality of optimal solutions and search efficiency. Test results show that FIPS is most effective in reducing the error in all the trials, while PSO starts by designating each position in the solution space as a potential design. Particle swarm optimizer (PSO) and advanced particle swarm optimization (APSO) can minimize the outage probability, which is used to find the optimal location for the receiver antenna.

In this work microstrip antenna is designed using particle swarm optimization (PSO) and advanced particle swarm optimization (APSO), investigated and compared.

\section{Particle swarm optimization}

Collective activities of a swarm of bees, fish, ant and other animals, the concept of particle swarm optimization (PSO) has been established and introduced into antenna design applications recently by Robinson and Rahmat-Samii [8]. 
Fathi [9] implemented PSO to improve the radial basis function. PSO is a population-based optimization method first proposed by Eberhart and Colleagues. The principle of PSO is based on the principle that each solution can be represented as a particle (agent) in a swarm. Each agent has a position and velocity vector and each position coordinate represents a parameter value. Thus for an $\mathrm{N}$-dimensional optimization, each agent will have a position in N-dimensional space that represents a solution. PSO also requires a fitness evaluation function that takes the agent's position and assigns to it a fitness value. The position with the best fitness value in the entire run is called the global best ( $\left.\mathrm{g}_{\text {best }}\right)$. Each agent also keeps track of its best fitness value. The location of this value is called its personal best ( $\left.p_{\text {best }}\right)$. Each and every agent is initialized with a random position and a random velocity. The velocity and position update of the swarm are expressed by following matrix equations:

$$
V_{\text {updated }}=V_{\text {previous }}+c_{1} \eta_{1}(P-X)+c_{2} \eta_{2}(G-X),
$$

where $\eta_{1}$ and $\eta_{2}$ are the uniform random variable in the range $0 \leq \eta \geq 1$ and $V$ previous is the initial velocity of the velocity at the beginning of the iteration. $P-X$ is the distance of the particle from the personal best position. $G-X$ is the distance from the global best position. In the early stages of the process, velocities are likely to have large values and will become substantially smaller as the swarm converges to optimum. For each and every iteration, the movement of particle is in the direction identified by the velocity matrix and according to a simple relationship given by

$$
X_{\text {updated }}=X_{\text {previous }}+V \Delta t .
$$

Flow chart depicting the PSO algorithm is given in figure 1.where $\mathrm{g}_{\text {best }}=$ global best position, $\mathrm{p}_{\text {best }}=$ self-best

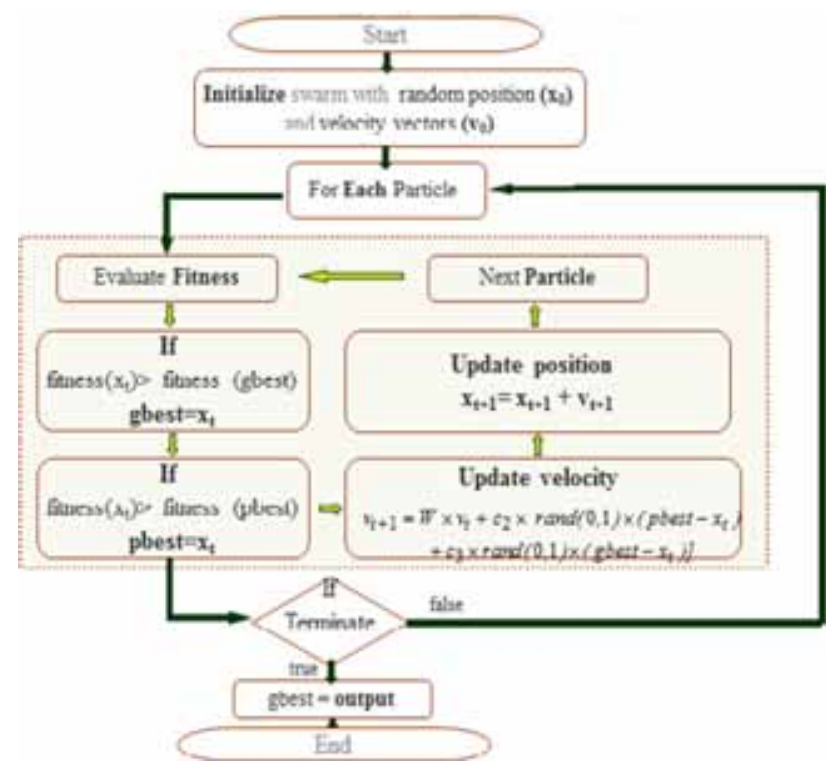

Figure 1. Flow chart of PSO algorithm.

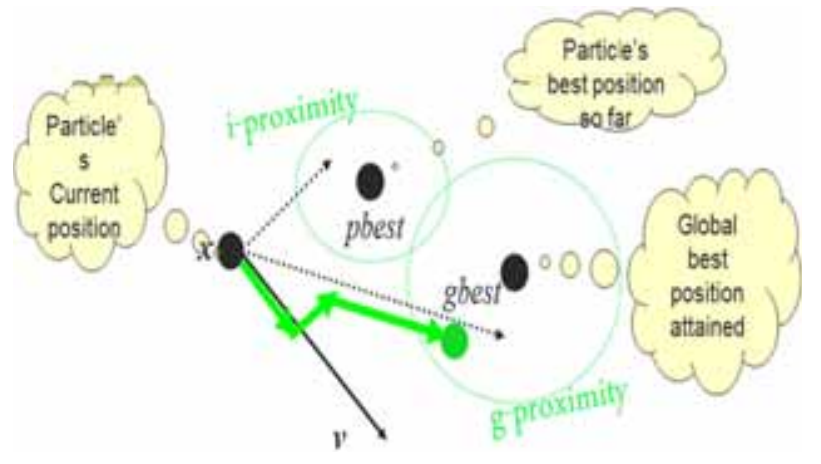

Figure 2. Psychosocial cooperation of particle position.

position, $c_{1}$ and $c_{2}=$ acceleration coefficients/weighting factors are used to control the impact of the local and global components in velocity equation, $W=$ inertial weight.

Psychosocial cooperation of particle position in PSO is shown in figure 2.

\section{Advanced particle swarm optimization}

The significant distinction between advanced PSO and a normal PSO is based on the population updating mechanism. All the particle velocities and positions are updated by PSO algorithm by using Eq. (3) at the end of the generation. Then it updates the best positions, $\mathrm{Xg}_{\text {best }}$ and $\mathrm{Xp}_{\text {best }}$.

Otherwise, the advanced PSO has updating mechanism that the new best position is found after each particle position is updated if the best position is better than the current best position. The new best position will be used in the following particle swarm immediately. The swarm reacts more quickly to speed up the convergence because the updating occurs immediately after objective function evaluation for each particle. APSO algorithm compared to the original PSO algorithm, APSO updates the particle velocity in a different and more sophisticated manner. It is NP Hard problem.

The particle swarm optimization (PSO) algorithm has been presented to meet rapidly during the initial stages of a global search, but around global optimum, the search will become very slow. Because of this reason, the mutation scheme is introduced in this algorithm to speed up the convergence when the particles are around global optimum. The mutation scheme also avoids premature convergences in the searching procedure and helps $\mathrm{Xg}_{\text {best }}$ to escape from the local optimal position.

PSO based search process is defined as follows:

$$
\begin{aligned}
v_{t+1}= & W \times v_{t}+c_{1} \times \operatorname{rand}(0,1) \times\left(p_{\text {best }}-x_{t}\right)+c_{2} \\
& \times \operatorname{rand}(0,1) \times\left(g_{\text {best }}-x_{t}\right)
\end{aligned}
$$

The random numbers in Eq. (3) are computed as 


$$
\begin{aligned}
& \text { random } 1=1-\left(F_{\text {bestid }} / F_{\text {id }}\right)+\text { random } \\
& \text { random } 2=1-\left(F_{\text {bestGd }} / F_{\text {id }}\right)+\text { random }
\end{aligned}
$$

where $F_{\text {bestgd: }}$ global optimal solution, $F_{\text {bestid: }}$ local optimal solution of current particle, $F_{\text {id }}$ : solution of current particle, random: a random number from a distribution $\mathrm{U}(0,1)$.

The approach of generating rand1 and rand 2 hints to different velocities in APSO. The particle velocity in APSO is associated to the distance between the current particle solution and global optimal solution. When the particle is far from the global optimal solution, its moving velocity in APSO is faster than the normal PSO. This change may accelerate the search process in some cases.

Search efficiency in optimization process and quality of optimal solutions are not satisfactory in PSO which is overcome by advanced PSO.

\section{Resonant frequency of dual slit-cut equilateral triangular patch}

The antenna is designed on a substrate of thickness $0.159 \mathrm{~cm}$ with substrate material being used is RT Duroid having $\varepsilon_{\mathrm{r}}=2.32$. The patch size is characterized by its side of equilateral triangle and is fed by a coaxial probe feed at position (Xr, Yr) as shown in figure 1. By introducing slots in microstrip structure dual frequency operation can be obtained. In this paper, an arrangement of PSO algorithm and IE3D simulation software is used to design dual band microstrip patch antenna, simulated and optimization results are presented [10]. Resonant frequency of the proposed antenna design is given as

$$
f_{\mathrm{ri}}=\frac{2 c}{P_{e} \sqrt{\varepsilon_{r}}}\left(m^{2}+m n+n^{2}\right)^{\frac{1}{2}}
$$

where $c$ is a speed of light, $P_{e}$ is the effective perimeter of modified triangular patch.

$$
\begin{gathered}
P_{e}=P_{i}+h\left(\varepsilon_{r}\right)^{-1 / 2} \\
i= \begin{cases}1, & P_{1}=3 a-\left(s_{1}+s_{2}\right)+2\left(s_{1}+b+c\right) \\
2, & P_{2}=3 a-\left(s_{1}+s_{2}\right)\end{cases}
\end{gathered}
$$

where $s_{1}$ and $s_{2}$ are slit width and $b$ and $c$ are slit length

$$
\begin{aligned}
v_{t+1}= & W \times v_{t}+c_{1} \times \operatorname{rand}(0,1) \times\left(p_{\text {best }}-x_{t}\right)+c_{2} \\
& \times \operatorname{rand}(0,1) \times\left(g_{\text {best }}-x_{t}\right)
\end{aligned}
$$

Microstrip antennas have good resonance at a particular frequency. The equations and constraints considered in this paper is integer linear programming. So from the designers' perspective resonant frequency optimization using PSO and advanced PSO are solution of the optimization problems in antenna design. The fitness function is defined using the

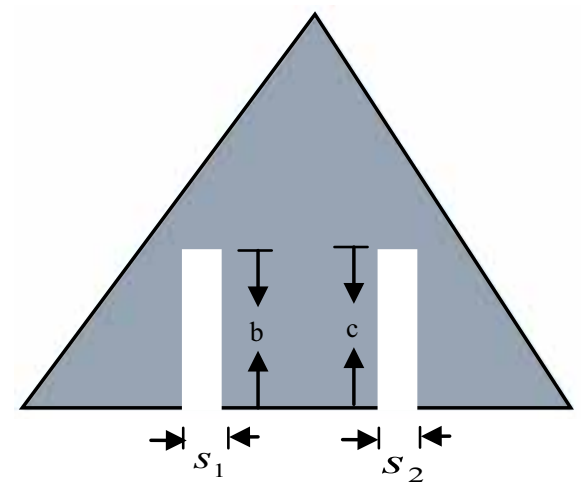

Figure 3. Top view of dual slit cut ETMA [10] () 2014 J. Inst. Eng. India Ser. B Springer, vol. 93.

input impedance expression for the aperture coupled microstrip antenna (figure 3).

\section{Application of particle swarm optimization (PSO) algorithm for micro-strip antenna}

PSO can be used in conjunction with some mathematical techniques for efficient antenna design as is discussed in [11-13]. Islam et al [14] and Vishnukanth and Raghavan [15] describe the particle swarm optimization to design microstrip antenna using curve fitting and G-shaped Monopole Antenna respectively. In this research work, antenna performance is optimized by PSO along with curve fitting. Zealand IE3D and MATLAB are used as simulation. By introducing slots in microstrip structure we may get dual frequency operation.

In this research work, the resonant frequency $f_{\mathrm{r}}$ of a dual slit cut equilateral triangular patch antenna (ETMA) is optimized using its input parameters like size of patch ' $a$ ', permittivity of substrate ' $\varepsilon_{\mathrm{r}}$ ', height of substrate h, Slit width $s_{1}=s_{2}=0.2 \mathrm{~cm}$, Slit length $b=c=2.0 \mathrm{~cm}$ and resonant frequency of patch taken as output.

PSO objective function is used to optimize the resonant frequency of dual slit cut ETMA and minimize the difference between measured resonant frequency value and the optimized resonant frequency value. PSO algorithm uses the number of agents -particles that constitute a swarm, moving around in the search space looking for best solution. Each particle modifies its position using following information:

- Existing position

- Existing velocities

- Distance between the existing position and $p_{\text {best }}$

- Distance between the existing position and gest

Mathematical model of modification of particle's position is given as 


$$
\begin{aligned}
v_{t+1}= & W \times v_{t+1}+c_{1} \times \operatorname{rand}(0,1) \times\left(p_{\text {best }}-x_{t}\right)+c_{21} \\
& \times \operatorname{rand}(0,1) \times\left(g_{\text {best }}-x_{t}\right) \ldots 7
\end{aligned}
$$

Following weight function is utilized in above equation

$$
W=W_{\max }-\frac{W_{\max }-W_{\min }}{\text { iter }_{\max }} \times \text { iter }
$$

where $W_{\max }$ : initial weight, $W_{\min }$ : final weight, iter $_{\max }$ : maximum iteration number, iter: current iteration number.

Using Eqs. (6) and (7), velocity, which slowly gets near to $p_{\text {best }}$ and $g_{\text {best }}$, is calculated. The current position (searching position) is modified.

Particle swarm optimizers generally require the engineer and scientists to select among a number of parameters before beginning the optimization. Here we are using the weighting factors $C_{1}$ and $C_{2}$ to both be 0.5 , initial weight of weight function $W_{\max }$ is 0.9 , final weight of weight function $W_{\min }$ is 0.4 , maximum iteration number itermax is 200 and population size is 100 . The complete algorithm was run with iteration of 100 . Then following coefficients values are obtained:

$$
\alpha_{1}=0.0342803429 \text { and } \alpha_{2}=0.918026171 \text {. }
$$

Resonant frequency of dual slit cut ETMA for different values of inputs are optimized.

\section{Application of advanced particle swarm optimization (APSO) algorithm for microstrip antenna}

Advanced particle swarm optimization (APSO) is extended method particle swarm optimization (PSO). Here particle exploits the information from all its neighbors to decide the next move rather than just the best one in PSO. In advanced particle swarm optimization (APSO) is determined as

$$
\begin{gathered}
V_{\mathrm{id}}=W \times\left(V_{i d}+\sum_{n=1}^{K i} C \times \operatorname{rand}() \times\left(P_{n b r n, d}-x_{i, d}\right)\right. \\
\text { If } V_{\text {id }}>V_{\max }, d, V_{\text {id }}=V_{\text {max }}, d \\
x_{i, d}=x_{i, d}+V_{\text {id }},
\end{gathered}
$$

where $K i$ is the number of neighbors that affects the particle velocity, nbrn the $n$th particle which affects the particle velocity and $C$ is weight value.

It is necessary to select the parameters value in order to produce best optimal solution. Each parameter value in PSO and APSO contains three levels in the experiments as shown in table 1.

We choose the population (number of particles) size 100. Initial values of these particles are randomly generated once and used. The search process terminates after 100
Table 1. Level of parameters in PSO and APSO.

\begin{tabular}{lc}
\hline Factors & Levels \\
\hline $\mathrm{W}$ & 0.9 \\
$C_{1}$ & 0.5 \\
$C_{2}$ & 0.5 \\
\hline
\end{tabular}

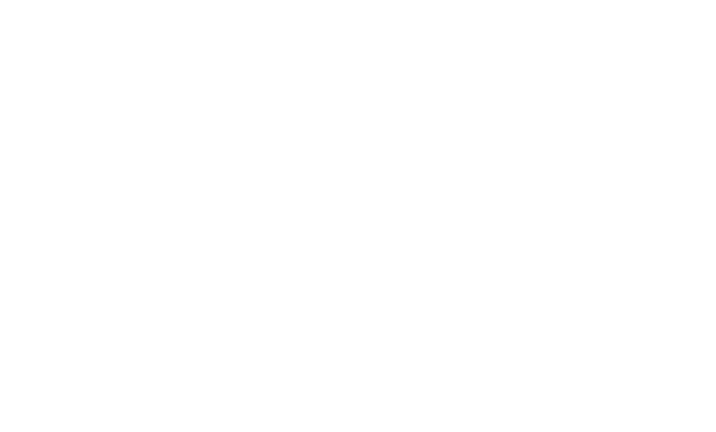

Figure 4. Minimized error generated by both algorithms on different test cases when size of patch ' $a$ ' is $4.1 \mathrm{~cm}$.

iterations. The minimized errors of both algorithms are shown in figure 4 . This figure shows that APSO produces the smallest error in most test conditions. It is concluded that APSO is more effective in optimization of dual slit-cut ETMA. The results also show that the resonant frequencies to a large extent influence the performance of the optimization algorithms, particularly PSO and APSO. The smallest error emin can be generated from these algorithms. We define ratio of error as

$$
\text { Ratio of error }=(\text { err }- \text { emin }) / \text { emin, }
$$

where err is the minimized error obtained by each run.

\section{Results and conclusion}

In this paper, the resonant frequency $f_{\mathrm{r}}$ of a equilateral triangular patch antenna is optimized using its input parameters like size of patch 'a', permittivity of substrate $\varepsilon_{\mathrm{r}}$, height of substrate $h$, and resonant Slit width $s_{1}=s_{2}=0.2 \mathrm{~cm}$, Slit length $b=c=2.0 \mathrm{~cm}$ is common and resonant frequency of patch taken as output. The data collected using proposed algorithm (APSO) in MATLAB is compared with theoretical and experimental results [4-8] as shown in table 2 .

A brief analysis of research on microstrip antenna design using PSO has been presented in the limited scope. The review shows that advanced PSO can also be used to overcome its inherent disadvantage of narrow bandwidth of ETMA. Search efficiency in optimization process and quality of optimal solutions are satisfactory in APSO compared to PSO. Therefore, this research work aims at 
Table 2. Proposed algorithm (APSO) optimized, simulated and theoretical values of resonant frequency of dual slit-cut ETMA.

\begin{tabular}{|c|c|c|c|c|c|c|c|}
\hline$a$ & $h$ & $\varepsilon_{\mathrm{r}}$ & $f_{\mathrm{r}}($ simulated $)(\mathrm{Hz})$ & $f_{\mathrm{r}}($ theoretical $)(\mathrm{Hz})$ & $f_{\mathrm{r}} \mathrm{CRO}(\mathrm{Hz})$ & $f_{\mathrm{r}}(\mathrm{ACRO})(\mathrm{Hz})$ & $\%$ Error of APSO \\
\hline 4.1 & 0.07 & 10.5 & 4.76 & 5.29 & 4.29 & 4.68 & 0.39 \\
\hline 4.1 & 0.06 & 10.5 & 4.98 & 5.26 & 6.26 & 6.96 & 0.7 \\
\hline 4.1 & 0.09 & 10.5 & 5.01 & 5.30 & 5.90 & 6.30 & 0.4 \\
\hline 4.1 & 0.08 & 10.5 & 5.00 & 5.32 & 6.32 & 6.89 & 0.57 \\
\hline 8.7 & 0.078 & 2.32 & 1.28 & 1.15 & 1.15 & 2.10 & 0.95 \\
\hline 8.7 & 0.080 & 2.32 & 1.55 & 1.75 & 1.75 & 1.79 & 0.04 \\
\hline 10 & 0.159 & 2.32 & 1.011 & 0.974 & 0.974 & 1.074 & 0.1 \\
\hline 10 & 0.150 & 2.32 & 1.031 & 1.0 & 1.02 & 1.75 & 0.73 \\
\hline
\end{tabular}

improving optimization of design of dual slit-cut ETMA by advanced PSO algorithms. This creates a solution space in the optimization larger than that in previous revisions. PSO and APSO algorithms have been used to search for optimal paths in the enlarged space. Completely factorial experiments were conducted to determine best parameter settings in these algorithms. Authors also investigate the performance of these two algorithms relating to different size of patch. The search capability of APSO is grander to PSO in a large solution space. This method can be effectively used in design of numerous multifaceted antennas. The performance of APSO in table 2 established the fact that the resonant frequency value optimized by proposed algorithm APSO has minimal error which is less and achieved results for resonant frequency are in good manner for design point of view. For further development researcher may employ two heuristic-based greedy construct algorithms [14] and compare the results with APSO algorithm to improve resonant frequency by minimizing the error ratio.

\section{References}

[1] Saunders Simon R 2003 Antennas and propagation for wireless communication systems. John Wiley and Sons Ltd

[2] Deschamps G A 1953 Microstrip microwave antennas. Proceedings of 3rd USAF Symposium on Antennas, 1953

[3] Munson R E 1973 Single slot cavity antennas assembly, US Patent No. 3713162. January 23

[4] Wong K L and Pan S C 1997 Compact triangular microstrip antenna. IEEE 997 Electron. Lett. Online no. 19970332, 33(6)

[5] Vishwakarama R K, Ansari J A and Meshram M K 2006 Equilateral triangular microstrip antenna for circular polarization dual band operation. Indian J. Radio Space Phys. 35: 293-296
[6] Bahl I J and Bharta P 1980 Microstrip antenna. Artech House, Massachustts, USA

[7] Yahya Rahamat Samii, Denis Gies and acob Robinson 2009 Particle swarm optimization (PSO): A novel paradigm for antenna designs. International Conference on advances in Computing, Control, \& Telecommunication Technologies, ACT '09. pp. 385

[8] Zhengyi Li and Ke Gong 2006 Design of a printed monopole antenna for Dualband WLAN application using PSO. 7th International Symposium on Antennas, Propagation and EM Theory. ISAPE'06. pp. 1

[9] Yogesh Choukiker, Behera S K, Mishra D and Mishra R K 2009 Optimization of dual band microstrip antenna using PSO. Applied Electromagnetics Conference (AEMC). pp. 1

[10] Malay Gangopadhyaya, Pinaki Mukherjee and Bhaskar Gupta 2009 The resonant frequency optimization of aperturecoupled microstrip antenna using particle swarm optimization algorithm. Applied Electromagnetics Conference (AEMC), pp. 1

[11] Verma K K and Soni K R 2005 Theoretical study of twoelement array of equilateral triangular patch microstrip antenna on ferrite substrate. Pramana J. Phys. Indian Acad. Sci. 65(3): 732-735

[12] Chung K L and Tam W Y 2008 Particle swarm optimization of wideband patch antennas. Asia-Pacific Microwave Conference, 2008. APMC. pp. 1-4

[13] Prabal Pratap, Bhatia R S and Binod Kumar 2014 Calculation of resonant frequency for a dual-slit cut equilateral triangular microstrip antenna. J. Inst. Eng. (India): B, 95(3): 185-188

[14] Islam Mohammad Tariqul, Misran Norbahiah, Take Tan Chuen and Moniruzzaman Mohd. Moniruzzaman, Optimization of microstrip patch antenna using particle swarm optimization with curve fitting. International Conference on Electrical Engineering and Informatics, 2009. ICEEI 'O9. vol. 02 , pp. 711-714

[15] Vishnukanth K Pavana and Raghavan S 2008 Design of an optimal G-shaped monopole antenna using particle swarm optimization. Proceedings of the 38th European Microwave Conference, pp. 389-342 